\title{
Percepciones de futuras educadoras de párunlos hacia la educación ciudadana
}

Perceptions of Future Early Childhood Educators about Citizenship Education

Artículo de investigación | Research article

Fecha de recepción: 11 de junio de 2020

Fecha de aceptación: 08 de junio de 2021

Fecha de disponibilidad en línea: noviembre de 2021

doi: 10.11144/Javeriana.m14.pfep

Olga Aurora Carrillo-Mardones

ocarrillo@uct.cl

Universidad Católica de Temuco, Chile

ORCID: http://orcid.org/0000-0003-3130-2047

Sixtina Pinochet-Pinochet

sixtina.pinochet@ucn.cl

Universidad Católica del Norte, Chile

ORCID: https://orcid.org/0000-0002-9252-5669

Carlos Muñoz-Labraña

carlosem@udec.cl

UniversidAd de Concepción, CHILE

ORCID: http://orcid.org/0000-0003-2044-1965

Dorama Elisa Leal-Burgos

dleal@uct.cl

Universidad Católica de Temuco, Chile

ORCID: http://orcid.org/0000-0002-0765-1901

Para citar este artículo | To cite this article

Carrillo-Mardones, O. A., Pinochet-Pinochet, S., Muñoz-Labraña, C. \& Leal-Burgos, D. E. (2021). Percepciones de futuras educadoras de párvulos hacia la educación ciudadana. magis, Revista Internacional de Investigación en Educación, 14, 1-26. doi: 10.11144/Javeriana.m14.pfep 


\title{
Resumen
}

Se presentan los resultados de una investigación realizada con estudiantes en práctica profesional de la carrera de Educación Parvularia en una universidad de la Araucanía, Chile. El objetivo fue indagar las percepciones de educadoras en formación sobre el desarrollo de la noción de ciudadanía en la educación infantil. La investigación, cualitativa e interpretativa, se realizó mediante entrevistas semiestructuradas a las estudiantes que efectuaban prácticas pedagógicas en jardines infantiles y escuelas rurales y urbanas de la región. Los resultados evidencian una falta de claridad en el tema, que repercute en las estrategias para abordarlo, lo cual hace prevalecer una visión normativa de la educación ciudadana.

\section{Palabras clave}

Percepción; educación ciudadana; primera infancia; docente de preescolar

\begin{abstract}
This paper presents the results of a research carried out with students in professional practice of the Early Childhood Education Career at a university in Araucanía - Chile. The objective was to investigate the perceptions of students of education about the development of citizenship in early childhood education. The research followed qualitative and interpretive research methods. We conducted semi-structured interviews with the students who carried out pedagogical practices in kindergartens, rural and urban schools in the region. Results showed a lack of clarity on the subject, which affects the strategies to address it, this makes a normative vision of citizenship education prevail.
\end{abstract}

\section{Keywords}

Perception; civic education; early childhood; preschool teachers 
Descripción del artículo | Article description

El artículo de investigación es parte del proyecto

Apreciaciones de la enseñanza de la ciudadanía en educación parvularia. Una mirada desde las educadoras en formación de la Universidad Católica de Temuco, desarrollado en torno a las apreciaciones de la enseñanza de la ciudadania en estudiantes en práctica profesional de educación parvularia. Este proyecto ha sido financiado por fondos internos de la Universidad Católica de Temuco y es dirigido por la Doctora Olga Carrillo Mardones de la Facultad de Educación de dicha casa de estudios.

\section{Introducción}

La educación ciudadana ha tenido un inmenso interés de investigación en el mundo, y estudios recientes realizados por la OCDE ${ }^{1}$ evidencian la relevancia que posee para los procesos de construcción democrática, así como para la adquisición de las competencias que les permitan a las personas incluirse y desenvolverse en sociedad.

A partir de lo señalado, dichas investigaciones comienzan a tener relevancia en América Latina, particularmente en Colombia, lo cual se vincula a los procesos de paz en dicho país (Díaz \& Rojas, 2018). Por otra parte, en Argentina y Chile se le ha prestado especial atención a la temática durante los últimos quince años. En Chile, específicamente, se relaciona con el aumento de la abstención en los procesos de participación política formal y la escasa valoración que las nuevas generaciones le asignan a la democracia (ICCS, 2016, en Agencia de la Calidad, 2018). El informe emanado del Consejo Asesor Presidencial que se organizó en Chile durante el año 2015 dejó en evidencia esto, así como también el rol que le corresponde a la educación, tanto en espacios escolares como en la misma sociedad, en cuanto a la generación de una cultura ciudadana democrática.

En respuesta a lo anterior, en el año 2016 se promulgó la Ley 20911 de formación ciudadana, que convoca a todo el sistema escolar a ser parte y responsable de dicha educación, y que obliga a los centros educativos a elaborar planes de formación ciudadana en los que se evidencie una formación con sentido democrático y participativo.

Considerando que la educación ciudadana constituye un eje fundamental para la sostenibilidad de una sociedad en la que se desarrolle y fortalezca la equidad, la justicia social, el respeto y la responsabilidad, corresponde a la escuela forjar las mejores posibilidades para su desarrollo, a

1 http://archivos.agenciaeducacion.cl/Educacion_en_Chile_OCDE_Nov2017.pdf 
través de oportunidades de aprendizaje y de experiencias para la vida democrática, de modo que las personas adquieran lo necesario para participar en la sociedad y logren reconocerse como parte de una comunidad (Bolívar, 2007; Gimeno, 2002; Orellana \& Muñoz, 2019; Peña, 2015; Redón, 2010). Asimismo, es importante que se les prepare para la vida pública, social y política, y que internalicen aprendizajes que favorezcan la participación responsable (Ley General de Educación [LGE], 2009; Ley 20911, 2016).

Es relevante entonces que esta educación se entienda como una herramienta para que niños, niñas y jóvenes se conciban como ciudadanos activos y responsables, con una visión del presente y futuro que incorpore aspectos ambientales, sociales y multiculturales (Bolívar, 2016; Maiztegui, 2007; Pagès \& Santisteban, 2009). A partir de lo señalado, y de acuerdo a lo prescrito en el artículo 12 de la Convención de los Derechos del Niño, la escuela contribuiría a hacer realidad los ideales según los cuales los niños "tienen derecho a expresar su parecer cada vez que toman decisiones que tienen que ver con ellos", y los adultos deben "tener en cuenta las opiniones de los niños". Dicho de otra manera, ella propicia que la educación en general y, la educación ciudadana en particular, se convierta en una suerte de caja de resonancia de la expresión de los infantes y de sus derechos.

Retomando lo planteado, y dada la relevancia e interés político y educativo que concita la implementación de la Ley 20911 de formación ciudadana al incluir esta enseñanza desde la educación inicial, la presente investigación ha estudiado las percepciones acerca de la educación ciudadana de las educadoras en formación que se encontraban, en el año 2017, en práctica profesional, a partir de una mirada crítica de lo que fue su propia formación, bajo las Bases Curriculares del nivel (MINEDUC, 2001), y de sus prácticas pedagógicas frente a niños y niñas, tal y como se consideraron en el plan de estudio. Esto porque, a nuestro parecer, comprender los procesos de enseñanza y aprendizaje que se están ofreciendo en la educación inicial resulta una tarea fundamental, pues lo que se realice o eventualmente deje de hacerse allí repercute en las etapas posteriores.

\section{La formación ciudadana en educación infantil}

El ejercicio de la ciudadanía, desde la infancia, requiere de compromiso por parte de las personas adultas, pues esto garantiza el derecho de participación y de aprendizaje de la ciudadanía a niños y niñas, con el fin de que puedan seguir construyéndolo durante todos sus ciclos de vida (Arrúe \& Consoli, 2010).

Investigaciones realizadas en la última década evidencian la relevancia que posee la educación infantil para potenciar los aprendizajes y el 
desarrollo de la personalidad en los infantes, debido a que son fundamentales en estos procesos las formas de enseñanza, así como la familia y la institución educativa (Osoro-Sierra \& Castro-Zubizarreta, 2017; Redón, 2010; Rodríguez et al., 2016). De acuerdo a la literatura, la institución educativa cumple un rol central si se quiere potenciar el desarrollo temprano de aquellas aptitudes ciudadanas que son necesarias para la vida democrática y la inclusión social (Graziella \& Rivera, 2016).

En este contexto, Nussbaum (2010) señala que la escuela puede contribuir a que niños y niñas desarrollen una conciencia del otro. Asimismo, les puede ayudar a comprender que es importante su preocupación por las necesidades y problemas que enfrentan los otros, y puede promover el abordaje de contenidos que permitan a las y los estudiantes llegar a una comprensión sobre la cultura del otro, para que, a través de esto, adquieran una posición empática frente a expresiones diversas.

Los estudios realizados por Redón (2010; 2019) plantean la importancia de la educación ciudadana en niños y niñas para configurar el sentido de lo cívico, de modo que el rol de los educadores resulta fundamental, pues están en constante relación con los infantes y conocen los contenidos conceptuales, procedimentales y actitudinales que implica el aprendizaje de la ciudadanía en ese nivel. Shuey \& Kankaras (2018) consideran que el aprendizaje temprano en los niños y las niñas tiene un impacto directo y duradero en logros educativos posteriores y en la participación cívica, lo cual resulta fundamental a la hora de favorecer prácticas de ciudadanía en la infancia que aporten a la comprensión y transformación democrática de la sociedad (Magendzo, 2008; Ruiz et al., 2016).

Por lo anterior, comprendemos que la participación, el ejercicio de la ciudadanía en democracia es un aprendizaje, y por ello es relevante que los niños y las niñas en educación infantil sean convocados a ser actores en este proceso, ya que estos "en la mayoría de las escuelas, no son invitados a identificar o definir sus intereses colectivos, mucho menos a expresar sus opiniones o participar en la toma de decisiones que, ciertamente, los afectan" (Arrúe \& Consoli, 2010, p. 3). A partir de lo expuesto, no nos cansaremos de insistir en que la enseñanza y el aprendizaje de la ciudadanía requiere ser realizada desde la primera infancia, pues posibilita la transmisión de aquellas virtudes cívicas que resultan imprescindibles y permiten la vida común (Bolívar, 2016). Asimismo, Moliner et al. (2016) expresan que la educación democrática bajo este expediente tendría un doble sentido: por una parte, educar para la democracia y, por otro, ejercerla activamente, siendo un fin y un medio al mismo tiempo.

La construcción de la ciudadanía es así una de las finalidades de la educación, ya que por medio de esta se pueden formar personas íntegras 
y responsables, que logren convivir de manera activa, propositiva e inclusiva, de modo que el otro/a se piense como un legítimo otro (Muñoz et al., 2019). Por ello, estar preparado para el ejercicio de la ciudadanía y reconocerse como parte de ella constituye una tarea fundamental para la cual se debe tener disposicion de trabajo, partiendo desde el elemento político hasta el educativo. De esta manera se pueden generar los cambios que se precisan en dicha materia (Delors, 1996), que refuerzan la comunidad y en cuyo papel es relevante el desarrollo de valores que potencien en los niños y las niñas la justicia, la solidaridad, el diálogo y el respeto (Cortina, 1997).

En relación con lo anterior, las Bases Curriculares de Educación Parvularia en Chile (MINEDUC, 2001; 2018) declaran ocho principios pedagógicos: bienestar, actividad, singularidad, potenciación, relación, unidad, significado y juego que, enmarcados en una propuesta humanista, potencian a niños y niñas como personas que aprenden confiadamente y con plena capacidad. En esta línea, la educación ciudadana se instala en el ámbito de formación personal y social, en el núcleo de convivencia (MINEDUC, 2001) y, con la actualización del año 2018, en el de convivencia y ciudadanía (MINEDUC, 2018).

\section{La enseñanza y aprendizaje de la ciudadanía en la infancia}

La experiencia que puede brindar el aprendizaje de la ciudadanía en el aula de educación infantil constituye un elemento relevante para la socialización política y para "la configuración del niño y la niña como sujetos de derechos" (Redón, 2019, p. 1), así como también para la adquisición de habilidades de reflexión y diálogo que promueven el sentido de pertenencia y de construcción de la vida comunitaria.

De este modo, un sistema educativo de calidad es clave para el aprendizaje de la vida en democracia, pues promueve la participación, el respeto y la tolerancia para el ejercicio de una ciudadanía que impulse la inclusión social y genera las condiciones para ello (Gimeno, 2002). Educar para la ciudadanía implica promover oportunidades desde la escuela y hacia ella, capacitando para reflexionar, así como para garantizar y fortalecer los valores democráticos (Bolívar, 2007; Carrillo \& Jurado, 2017). Así, a través de este expediente, se constituye una herramienta fundamental para que los niños y las niñas se conciban como parte de una ciudadanía activa y participativa, en la que son capaces de interactuar en comunidad (García \& De Alba, 2014; Graziella \& Rivera, 2016; Martínez \& Cumsille, 2015).

La educación ciudadana es responsabilidad de todo el sistema educativo y, por tanto, se debe abordar desde los primeros años de esta de manera transversal. El propósito es que niños y niñas reciban, de manera 
temprana y permanente, una formación que incentive su participación en la vida democrática. Lo anterior es coherente con el principio 10 de la Proclamación de los Derechos del Niño (ONU, 1959), el cual plantea que todo niño o niña "debe ser educado en un espíritu de comprensión, tolerancia, amistad entre los pueblos, paz y fraternidad universal, y con plena conciencia de que debe consagrar sus energías y aptitudes al servicio de sus semejantes".

En ese sentido, Trilla \& Novella (2001) han investigado los distintos tipos de participación infantil que se generan en los ambientes educativos escolares, y han logrado establecer cómo estos inciden en el desarrollo de niños y niñas como agentes que asumen su protagonismo, tanto en la construcción de sus aprendizajes como en sus intervenciones en tanto sujetos sociales. Al respecto, los autores destacan la necesidad de que, desde las escuelas, se transite a la promoción de una participación de tipo proyectiva, en la que el estudiantado asuma un rol protagónico con un mayor compromiso y corresponsabilidad, lo cual implica el ejercicio de una participación real, significativa y que contribuya al desarrollo de competencias para la misma.

En la misma línea, Pinochet (2015) propone que la escuela sea una institución donde se viva la ciudadanía activa, en la que niños y niñas sean formados reconociendo sus particularidades, siendo actores principales, promoviendo la iniciativa personal, la igualdad, la solidaridad y su sentido crítico. Por ello, construir una sociedad inclusiva, equitativa y justa requiere de procesos de transformación social basados en la participación real, efectiva y democrática, en el que las personas y los grupos puedan participar en condiciones de igualdad (Mata \& Ballesteros, 2011).

Por ello, la escuela es el escenario donde se debe invitar a los niños y las niñas a expresar sus opiniones, a participar en la toma de decisiones, a ser los protagonistas de su constitución como ciudadanos y ciudadanas a partir de sus concepciones y de sus propias experiencias, siendo importante en este proceso la mediación de las educadoras para fortalecer valores para la vida en comunidad (Moliner et al., 2016). Con el fin de lograr lo antes expuesto, se deben desplazar las visiones adultocéntricas, que limitan la ciudadanía a un aprendizaje asimétrico tensional (Figueroa, 2016), y que excluyen a niños y niñas de su propia participación en los procesos de construcción y formación de la ciudadanía, subordinando la infancia a la concepción adulta de esta. En palabras de Trilla y Novella (2011): "Solo en la medida en que los niños puedan ejercer genuinamente su derecho a participar será realmente eficaz la formación para la participación y la ciudadanía" (p. 32). 


\section{La formación inicial docente y la ciudadanía}

La raíz que posibilita la sustentabilidad de la educación ciudadana ha de estar anclada en la formación inicial de las educadoras. Lo anterior viene determinado por las experiencias formativas que se experimenten en estos procesos, puesto que serán relevantes para que los y las docentes en formación permitan a niños, niñas y jóvenes construirse como sujetos reflexivos, favoreciendo su aprendizaje social, así como la adquisición de aquellas capacidades sociales y normas que permiten la convivencia entre los grupos (Gil-Madrona et al., 2016). De esta manera, la formación inicial de la educadora debe orientarse para garantizar a los infantes aprendizajes que promuevan experiencias de ciudadanía para la vida cotidiana, de la mano con los principios que guían la educación parvularia (Ley 20903, 2016; MINEDUC, 2008; 2012).

También, es fundamental considerar las experiencias de formación práctica de las educadoras, que facilitan la articulación de la teoría y la práctica en un contexto real, permitiéndoles diseñar proyectos de aprendizaje (Tejada-Fernández et al., 2017). Todo esto constituye un momento clave en los procesos formativos, al favorecer la integración de los saberes en los diseños de enseñanza, en un contexto específico de realización (CastroZubizarreta \& García-Ruiz, 2016).

En esta línea, las experiencias de innovación pedagógica en formación ciudadana (MINEDUC, 2018) evidencian la importancia de trabajar de manera articulada entre las asignaturas, pues esto potencia la participación consciente, activa, reflexiva y crítica en el proceso, tanto para el estudiantado como para la comunidad educativa en general, reforzando valores como el respeto a la diversidad, lo que orienta a la realización de experiencias significativas de educación ciudadana en la infancia.

Por lo anterior, y considerando que es necesario abordar la educación ciudadana desde las primeras etapas formativas del ser humano, es fundamental la participación temprana de las educadoras en este proceso. Pero, ¿cómo se está desarrollando este proceso en nuestro país? ¿Las educadoras que realizan su práctica profesional incorporan la enseñanza de la ciudadanía en su trabajo?; y si lo hacen, ¿cuáles son las estrategias utilizadas por estas profesionales?

Al plantear tales interrogantes, además de hacernos partícipes de la preocupación por esta importante cuestión, hemos creído conveniente avanzar en ella, indagando las percepciones que poseen las educadoras en formación acerca de la enseñanza de la ciudadanía en la niñez, investigando continuamente las estrategias utilizadas por estas profesionales en el nivel de educación parvularia (3 meses a 6 años de edad). 


\section{Metodología}

La investigación utiliza un enfoque cualitativo e interpretativo, pues la idea es comprender e interpretar el fenómeno de estudio desde los significados de las propias educadoras, considerando sus percepciones, intenciones y acciones (Hernández et al., 2006; Pérez, 2008). Con un enfoque cualitativo, la investigación opta por un estudio de caso instrumental que no busca la generalización, sino la comprensión de un fenómeno concreto de interés y poco indagado (Coleman \& Unrau, 2005; Stake, 2007), como lo es el conjunto de percepciones que poseen las futuras educadoras de párvulos hacia la educación ciudadana.

El estudio comprendió una indagación con siete educadoras en formación inicial. Los criterios de selección fueron: haber realizado la práctica profesional durante el año 2017, haber tenido esta experiencia formativa en algunos de los jardines infantiles rurales y/o urbanos dependientes del Estado y que los jardines en que se realizaron esas prácticas profesionales estuvieran ubicados en algunas de las comunas de la región de La Araucanía, Chile.

Tabla 1.

Identificación de las educadoras en formación entrevistadas

\begin{tabular}{l|l|c}
\hline $\begin{array}{l}\text { Identificación de } \\
\text { la educadora en } \\
\text { formación inicial }\end{array}$ & $\begin{array}{l}\text { Establecimiento educativo } \\
\text { donde se desempeñaron }\end{array}$ & $\begin{array}{l}\text { Semestre y año de } \\
\text { realización de la } \\
\text { práctica profesional }\end{array}$ \\
\hline Educadora en formación 1 & Escuela urbana 1, comuna de Temuco & I- 2017 \\
\hline Educadora en formación 2 & Escuela urbana 2, comuna de Padre las Casas & I- 2017 \\
\hline Educadora en formación 3 & I- 2017 \\
\hline Educadora en formación 4 & Escuela rural 1, comuna de Padre las Casas & I- 2017 \\
\hline Educadora en formación 5 & Escuela rural 2, comuna de Padre las Casas & I- 2017 \\
\hline Educadora en formación 6 & Jardín infantil urbano 1, comuna de Temuco & II- 2017 \\
\hline Educadora en formación 7 & Jardín infantil urbano 2, comuna de Temuco & II- 2017 \\
\hline
\end{tabular}

Fuente: elaboración propia.

Los datos fueron obtenidos a partir de siete entrevistas semiestructuradas. El procesamiento de la información se realizó en tres etapas. La primera incluyó la revisión de la literatura especializada. La segunda contempló la realización de las entrevistas semiestructuradas y su posterior transcripción, seguida por el establecimiento de los primeros focos del análisis mediante una lectura y relectura del material empírico disponible, constituido por una masa textual de 26000 palabras aproximadamente. Lo anterior condujo a la identificación de las primeras unidades comprensivas del problema de investigación (Stake, 2007; Strauss \& Corbin, 2002). En la tercera etapa analizamos formalmente la información, subrayando, preguntando, conjeturando, reduciendo los datos y seleccionándolos para hacerlos 
significativos, abarcables y manejables, utilizando para ello el análisis de contenido y la ayuda del programa ATLAS.ti. A partir de este material, se depuraron las dimensiones del problema de investigación, se denominaron las unidades de análisis referidas a las categorías identificadas, se estableceron ciertas pautas de observación y, por último, se construyeron mediaciones analíticas entre las dimensiones y las categorías, procurando establecer su importancia y relación (Stake, 2010). Lo anterior fue acompañado de la enunciación de proposiciones narrativas encaminadas a describir cada categoría, lo que implicó una elaboración conceptual de la información recopilada. El peso del análisis descansó sobre el material simbólico de los datos, expresados a través del lenguaje propio de las educadoras en formación. Con el propósito de mantener y resguardar su identidad y opiniones, se acordó referenciarlos mediante una sentencia genérica que los identificara.

\section{Resultados}

A partir del análisis de los datos surgen tres categorías que permiten explicar el fenómeno en estudio.

Figura 1.

Categorías y subcategorías que emergen de las entrevistas

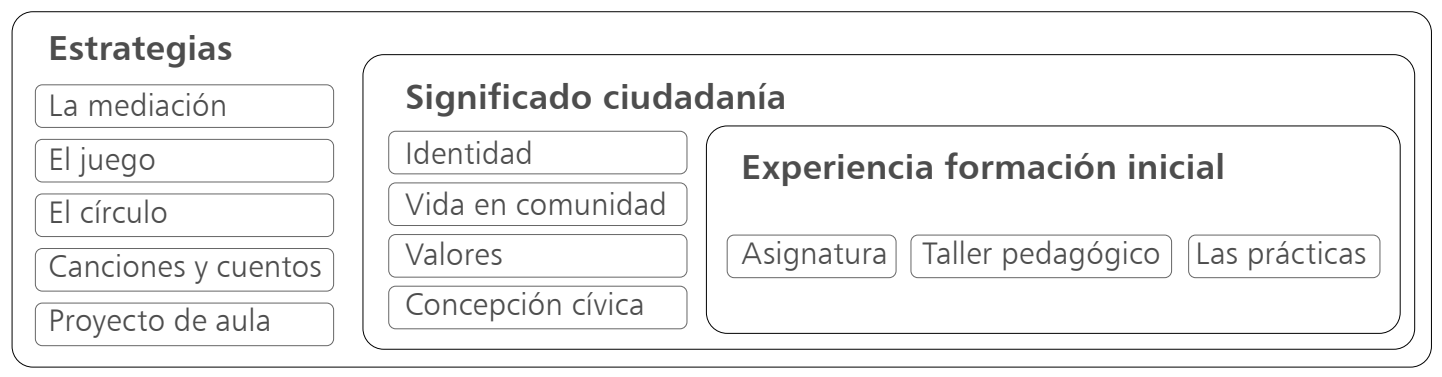

Fuente: elaboración propia.

Tabla 2.

Definición operacional de categorías

\begin{tabular}{l|l}
\hline $\begin{array}{l}\text { Experiencias de } \\
\text { formación inicial }\end{array}$ & $\begin{array}{l}\text { Consideramos en esta categoría los momentos formativos que las participantes } \\
\text { reconocieron como espacios para la formación ciudadana, que al mismo tiempo } \\
\text { les permitieron desarrollar las competencias pedagógicas para contribuir al } \\
\text { desarrollo de una cultura democrática y participativa en el contexto escolar. }\end{array}$ \\
\hline $\begin{array}{l}\text { Significado de } \\
\text { la educación } \\
\text { ciudadana }\end{array}$ & $\begin{array}{l}\text { En esta categoría se incluyeron las comprensiones y finalidades } \\
\text { que las participantes le asignaron a la educación para la ciudadanía } \\
\text { en el contexto escolar al nivel de educación parvularia. }\end{array}$ \\
\hline Estrategias & $\begin{array}{l}\text { Comprendidas como las acciones educativas que las participantes } \\
\text { utilizaron en el contexto escolar para promover actitudes y habilidades } \\
\text { ciudadanas entre niñas y niños. Se incluyeron estrategias y recursos } \\
\text { utilizados para contribuir al desarrollo de la ciudadanía. }\end{array}$ \\
\hline
\end{tabular}




\section{Experiencia de la formación inicial docente}

Se parte de la base formativa de las educadoras, aludiendo a los elementos que, desde lo transversal, disciplinar y pedagógico, contribuyeron en la incorporación de la educación ciudadana.

En esta categoría, las futuras educadoras hacen referencia a los elementos que tributan a la educación ciudadana, entre ellas la asignatura: "Lo que recuerdo es que nos hicieron un curso donde tenía que ver eso. Nos pasaron de lo que se trataba más o menos, esa vez nos dieron estrategias para trabajar con los niños" (Educadora en formación 3). No obstante lo anterior, en otras educadoras en práctica la asignatura no estuvo presente en su itinerario formativo: "No recuerdo que haya habido un ramo específico, como que tuviera el nombre de ciudadanía o formación ciudadana, no" (Educadora en formación 1).

En el caso de quienes tuvieron el curso señalan que: "Nos hablaban ahí en ese curso, fue el tema de dar, escuchar a los niños lo que tienen que decir. Y esas fueron prácticamente las dos cosas que se me quedaron de ciudadanía" (Educadora en formación 3).

A partir de la cita anterior, se refleja la poca claridad sobre la relevancia del tema. Además, coincide con aquellas que no tuvieron la asignatura: "No, no hay, pero sí se ve de forma transversal, seguramente en algún DDO o alguno del plan común, puede ser que haya habido, pero en verdad no lo recuerdo" (Educadora en formación 1).

Una docente establece una relación entre la asignatura y las Bases Curriculares:

Nos hizo ver estas otras situaciones que uno no se daba cuenta que estaban dentro de la formación ciudadana, pero generalmente en la práctica uno se guía netamente por las bases. Sí, este ramo me amplía situaciones, previsiones, pero uno (...) toma las bases y hace una actividad que sea acorde a la edad y, lúdica para los niños. (Educadora en formación 7)

Estos son elementos que, como sabemos, guían el diseño de los aprendizajes de los niños y las niñas en el aula.

Las entrevistadas también explicitan la falta de orientaciones y estrategias didácticas para abordar el tópico de la educación ciudadana con los niños y las niñas: "Que nos den estrategias para trabajarlo con los niños, yo creo que eso que nos enseñen, yo creo que eso faltó, faltó mucho" (Educadora en formación 1). Lo señalado, por un lado, contribuiría a facilitar el acercamiento de la enseñanza y el aprendizaje de la ciudadanía en educación infantil, y, por otro, evidencia la falta de una asignatura que diera las orientaciones: 
El que tenemos que enseñar a los niños que tienen que escucharse, en que los niños se pueden expresar, empezar a trabajar el tema de lo que se vive en sociedad como adulto, como por ejemplo el tema de votar, el mismo tema de lo que hay en las calles, señaléticas, como ese tipo de cosas más que nada se intenciona, pero una asignatura que hable de eso, no. (Educadora en formación 2)

Además de lo expresado anteriormente, también hay educadoras en formación que sitúan el contenido de ciudadanía en una experiencia formativa específica, como es el caso de un taller pedagógico: "Nos presentaron una ley que salió el año pasado que dice que hay que formar en ciudadanía a los niños de todos los niveles, de incluso pre básica y yo creo que ese taller pedagógico me enseñó bastante en relación a la ciudadanía" (Educadora en formación 4).

Por otro lado, a partir del discurso de las entrevistadas emergen algunas opiniones que revelan la importancia que han tenido las prácticas en los establecimientos educativos en los procesos que las han llevado a incorporar la educación ciudadana, aunque, como se ve, no con mucha claridad: "Es que lo que pasa es que todo fue como muy general, eso se fue aprendiendo más que nada en la práctica" (Educadora en formación 1).

\section{Significado de la formación ciudadana}

Esta categoría hace referencia a las apreciaciones que las educadoras en el estudio le otorgaron al significado de la educación ciudadana. Sobre el particular, las opiniones se agrupan, por un lado, bajo el tema de importancia para el desarrollo de la identidad, que puede ser refrendada en declaraciones como: "Es una parte de la identidad de los niños, es algo que tienen que aprender para cuando ya sean adultos y que la idea es que se vaya interiorizando desde pequeños" (Educadora en formación 3). Por otro lado, las opiniones se relacionan con algunos valores clave para vivir en sociedad, como se desprende de la siguiente afirmación: "Yo creo que serían niños mucho más responsables, mucho más conscientes de su rol dentro de la sociedad, de niños que trabajen de forma pacífica, niños más respetuosos, yo creo que por ahí va la cosa" (Educadora en formación 1).

Sin embargo, existen otras educadoras en formación que dieron cuenta de la relevancia de la educación ciudadana para la convivencia y la vida en comunidad:

La importancia de la formación ciudadana es que sin ella no podríamos ser una sociedad, no nos podríamos entender, no podríamos respetar la diversidad por ejemplo en los niños en este caso, la importancia radica, 
porque en primer lugar es la etapa como más marcadora para el niño y es donde empiezan ellos a relacionarse a compartir, aprenden por ejemplo a escuchar, a esperar el turno de habla, a expresarse, encuentro que en ese momento es como el momento decisivo donde podemos marcar al niño en cuanto a su formación en la sociedad. (Educadora en formación 2)

Por otro lado, encontramos educadoras en formación que relacionan la educación ciudadana con la participación: "Que, en todas las acciones, por ejemplo, el hecho de elegir un tema para poder exponerlo, eso ya es ciudadanía. El tener que desarrollar trabajos en equipo, ya es ciudadanía" (Educadora en formación 4). Así también con la alteridad como principio de comunidad:

Es como de que uno se sienta parte de estar con los otros, incluyendo que eso implica el respeto también por el otro, así yo veo la ciudadanía, que es sentirse parte de una comunidad o de un lugar y ser capaz también de convivir con ellos, poder respetarlos y conocerlos, y así lo veo yo. (Educadora en formación 5)

En un plano más institucional, las educadoras también sitúan a la educación ciudadana con los ámbitos propios que incluyen las Bases Curriculares para el nivel parvulario:

Yo lo relaciono mentalmente con el ámbito de convivencia, también el ámbito de formación personal y social, porque igual tiene que ver con reconocerse uno igual, cierto, nuestras capacidades, y también puede ser con el núcleo de grupos humanos, sus formas de vida y acontecimientos relevantes, por ello igual hay aprendizajes esperados que tienen que ver con el conocer las otras culturas y conocer otros lugares, así que con esos tres los relaciono yo, en las bases. (Educadora en formación 5)

Finalmente, existen otras educadoras que relacionaron la educación ciudadana con una concepción cívica de la ciudadanía, como se desprende de lo siguiente:

En los actos se ven muchas situaciones, por ejemplo, el hecho de cantar el himno nacional, también compartir qué están aprendiendo los otros niveles, saber comportarse en distintas áreas, por ejemplo, en actos escolares, los niños aprenden a comportarse, a escuchar, a estar tranquilos una cierta cantidad de tiempo, a escuchar a quien está hablando. Yo creo que en esa área está más presente la ciudadanía y la formación ciudadana. (Educadora en formación 4) 
Aquí podemos evidenciar que se destaca el valor de los símbolos patrios y el respeto a los actos presentes en las instituciones educativas.

Lo expuesto anteriormente nos hace reflexionar respecto a la falta de claridad por parte de las educadoras en formación en relación con el significado que tiene la educación ciudadana:

Ni yo tengo bien claro lo que es, entonces si yo no lo tengo claro, si yo no sé bien lo que es la formación ciudadana, a lo mejor yo sí la puedo practicar, pero si usted me pregunta yo no sé cómo explicárselo a usted, entonces si no sé describir lo que es, ¿cómo yo lo voy a enseñar a los niños? (Educadora en formación 1)

\section{Estrategias para la formación ciudadana}

Esta categoría incluye todo un conjunto de procedimientos y recursos utilizados por las educadoras para generar el aprendizaje de la ciudadanía en el nivel parvulario. En relación con ello, las educadoras exponen la estrategia de la mediación, pues esta les permitió mantener la convivencia en el aula:

A través de la mediación irles indicando qué está mal, qué está bien, o cómo hacerlo mejor, a enseñarles la empatía de aprender a escuchar al otro compañero, por ejemplo, siempre trabajo con que levanten la mano y que se escuche el compañero, entonces yo creo que ahí está, en eso. (Educadora en formación 3)

Otra de las estrategias que emerge del material empírico analizado, y que da cuenta de las estrategias utilizadas por las educadoras, corresponde al juego, destacando cómo a través de este los niños y las niñas adquieren aprendizajes:

Los niños aprenden a través del juego, para ellos todo lo que es lúdico los motiva, llama su interés. La idea es que durante la etapa preescolar ellos puedan jugar todo lo que se pueda y de ahí ir sacando aprendizajes, porque lo que nos enseñaron también, es que no solamente a través de un aprendizaje, por ejemplo, si quiero trabajar un núcleo y un aprendizaje de ese núcleo, si voy a hacer un juego que los niños compartan, un aprendizaje donde los niños compartan. (Educadora en formación 3)

Por otro lado, el juego también favorece la organización y el diálogo, sobre todo en aquellos que involucran a varios niños y, por lo tanto, tienen un carácter colectivo:

Los niños aprenden todo jugando, todo a través del juego y, bueno como le decía, el poder organizarse, en juegos grupales más que nada, porque 
los juegos que son más individuales yo creo que no mucho, por ejemplo, el poder consensuar, no sé, los roles o el que los niños vayan a jugar, a ver... a la tiña, por ejemplo, que ya el ponerse de acuerdo quién va a ser el que va a timar y quién va a correr o no, no sé, como eso... (Educadora en formación 1)

Continuando en la misma línea, no cabe duda de lo que quieren expresar las educadoras, el juego potencia las habilidades sociales:

Yo creo que el niño por medio del juego aprende a ser ciudadano, porque el juego yo creo que es como primordial, es importante para el niño porque es el momento en que socializa con sus compañeros, aprende a compartir. Puede aprender todo por medio del juego, porque el niño lo hace en forma espontánea. (Educadora en formación 4)

Una de las estrategias más utilizadas por las educadoras es el círculo, el cual puede ser abordado en diferentes actividades:

Los niños...son los niños, si bien yo les voy dando como la pauta para que ellos vayan expresándose, pero la idea es que ellos hagan, porque, a ver, por ejemplo, al momento de saludarse, iya!, los saludamos siempre a ellos, son todos ellos, después ellos me saludan a mí, yo les pregunto cómo están y son ellos los que están todo el rato participando, no es que yo me siente y solo hable yo...y ellos solamente me escuchen, como no sé, como siempre receptores, no, ellos son los que se expresan y ellos son los que trabajan el círculo, ellos son los que se cuentan, ellos son los que ven el tema de la asistencia, ellos recuerdan la fecha, el día, el mes, el año. (Educadora en formación 1)

En relación con lo anterior, se puede vislumbrar que el círculo posee varias funciones y permitió a la educadora abordar diversas temáticas:

Con el círculo uno puede trabajar muchas cosas, por ejemplo, podemos trabajar de matemáticas, podemos trabajar en lenguaje, podemos expresar, se pueden trabajar muchas cosas, pero yo creo que lo más importante es que es un momento donde nosotros podemos como reunirnos y conversar con el niño. (Educadora en formación 2)

Asimismo, el momento del círculo es el momento más valorado por las educadoras, pues en él se experimentan aprendizajes relacionados con el diálogo y la participación:

El círculo es el momento del saludo, el momento más importante de la jornada porque los niños en el círculo primeramente se expresan, los niños 
cuentan qué hicieron en sus casas, hablan, dialogan, por medio de preguntas uno les va preguntando, socializa con ellos, ellos socializan entre ellos también, desarrollamos canciones, tableros, por ejemplo, ellos recuerdan qué día es hoy, o también la fecha, el clima, o las normas también. (Educadora en formación 4)

Además, el círculo es una experiencia de relación horizontal y simétrica. Como señala una de las educadoras:

[...] un momento súper importante porque estamos todos en un mismo nivel, si nos sentamos todos en un círculo, nos miramos todos las caras, también se desarrollan hartas actividades, por ejemplo, el hecho de contar la asistencia; tributan a varios aprendizajes en sí, a la vez. ¿Cuál es la importancia? Desarrollo de varias áreas en forma integral en los niños. (Educadora en formación 4)

En esta misma línea, el círculo corresponde a una instancia de aprendizaje de valores y de ejercicio de participación democrática:

Yo creo que es el hecho de que ellos se puedan expresar, se pueden expresar libremente, ya, no hay, yo no los estoy guiando en su respuesta, ellos solo, ellos responden lo que ellos sienten, lo que ellos piensan, y también se les va, o sea, en cierto modo se les va guiando para que ellos también puedan respetarse, respetar las opiniones de los demás, el que respeten los turnos, en que levantan la mano y escuchen al compañero, la empatía se trabaja mucho en el momento del círculo. (Educadora en formación 1)

Además, destaca la relevancia del círculo al ser una de las estrategias que más se acerca a la educación ciudadana:

Creo que el círculo, yo creo que es como lo más, lo que más se trabaja la ciudadanía, por ejemplo... muchas veces se le hace elegir al niño, se le da por ejemplo la opción a que ellos puedan votar, yo por lo menos, algunas veces los hago votar por alguna canción o votar por algún cuento... ahí ellos pueden dar su opinión, van votando, de repente contamos los votos y ya ganó este otro, y también se le hace ver al niño por qué es importante respetar las opiniones de los demás. (Educadora en formación 2)

A la vez, el círculo, en el jardín infantil, entrega lineamientos a las educadoras para organizar la jornada con los niños y las niñas a partir de los relatos que emanan desde los propios infantes:

En general lo normal es que nosotras elaboremos toda nuestra temática del día con base en lo que en ese círculo conversamos o incluso lo 
que antes habíamos quedado de acuerdo, por ejemplo, levantar la mano cuando uno habla o respetar al compañero, escuchándolo, ese tipo de cosas son parte del círculo, por lo tanto, son aspectos de ciudadanía que uno trabaja o que uno va desarrollando en ese minuto. (Educadora en formación 6)

Por otro lado, el uso de canciones y cuentos emergió como estrategia utilizada por las educadoras para promover actitudes, enseñar valores o aprender vocabulario. En el caso de las primeras, se expresa que:

A través de las canciones ellos pueden aprender vocabulario también, se les puede enseñar quizás valores, dependiendo de la canción, por ejemplo, para el día de la familia tuvieron que aprender una canción de la familia, ¿y qué les enseña la familia? Que hay diferentes tipos de familia, que el papá puede estar separado de la mamá, pero igual lo quiere, que igual están para él. (Educadora en formación 3)

En relación a los cuentos, se manifiesta:

Una estrategia buena y que considero yo que es súper relevante tiene que ver con los cuentos, porque a veces en el cuento uno enseña historias y que para los niños igual son significativas, por ejemplo, o que tiene cierto aprendizaje esa historia, yo encuentro que ese es un buen, es una buena estrategia para poder enseñar a los niños la ciudadanía. (Educadora en formación 5)

También se menciona el proyecto de aula, el cual fomenta la educación ciudadana, pues permite la implicación de niños y niñas en la elección de los aprendizajes que les gustaría abordar y que son considerados por las educadoras:

Todo el proyecto de aula se va trabajando con lo que quiere el niño, no es algo que uno impone, porque los otros ejes centralizadores más que nada son temas que uno propone, uno realiza las experiencias, pero acá realmente el niño es como el protagonista, porque entre ellos llegan en conjunto a una conclusión: queremos hacer esto o ya se decidió esto, vamos a hacer esto, me gustaría hacer de esta forma, yo creo que esa es como la mayor, es el mayor trabajo que se hace con el tema de formación ciudadana. (Educadora en formación 2)

En relación con lo anterior, una de las entrevistadas expone:

A los niños yo les enseño de manera transversal lo que es ciudadanía, en el hecho de escoger, por ejemplo, el tema de un proyecto de aula, ellos 
primero hacen una votación, primero se les explica cómo hay que realizar la votación, hay que respetar al compañero, que aunque uno es amigo de otro no necesariamente tienen que tener la misma opinión o el mismo voto, hay que respetar también la opinión de la mayoría, también de la minoría. En relación a eso en forma transversal, pero no recuero muy bien qué aprendizaje esperado en específico tributan a la ciudadanía, pero sé que en ese núcleo se trabaja la ciudadanía de forma explícita. (Educadora en formación 4)

Por otra parte, es posible reconocer la utilización de varios recursos, entre ellos, los vídeos que potencian la enseñanza de normas en la sala:

Hay muchos videos para los niños, para enseñarles la consecuencia de sus actos, las normas. Yo creo que más bien eso, eso es lo que yo uso. [También utilizo] estrategias como de recreación, o juegos de roles también, dramatizaciones con los niños, también el uso de títeres, siempre es importante, porque por medio del diálogo finalmente se va enseñando; o sea, ellos lo han aprendido por medio del diálogo. (Educadora en formación 4)

Del análisis de las ideas expresadas por las educadoras, se identificó que estas utilizan prácticas asociadas al diálogo para generar un ambiente de participación democrático al interior de las aulas: "Bueno lo visualizo mucho en lo que es el diálogo, en lo que es hablar de los niños acerca de él, cuáles son sus intereses, cierto, cuando uno recoge los conocimientos previos que ellos tienen los va conociendo y sabiendo qué les interesa" (Educadora en formación 6). El diálogo para ellas corresponde a una estrategia de promoción de ciertos valores que favorecen el proceso de enseñanza y aprendizaje:

El que uno quiere enseñar algo, pero primero tiene que aprender a respetar, cuando habla el resto, o cuando uno quiere opinar, cómo reaccionar, o cuando alguno le gusta cómo decirlo... entonces eso se va trabajando constantemente... no es algo que sea, quizás se puede tomar como un tema, pero ese tema o ese se va trabajando siempre de manera transversal, lo que uno enseña. (Educadora en formación 7)

\section{Discusión de resultados}

En relación con las percepciones que poseen las educadoras en formación respecto a la ciudadanía en educación infantil, es interesante constatar cómo, una vez promulgada la Ley 20911 en marzo de 2016, las instituciones 
que forman estas profesionales procuraron paliar el eventual déficit formativo a través de talleres o charlas. Sin embargo, a pesar de esos esfuerzos, las estudiantes en su proceso final de formación evidenciaron falta de claridad al momento de identificar los conocimientos, las habilidades y las actitudes ciudadanas promovidas en este proceso. Esto resulta consistente con los hallazgos de investigaciones realizadas por Muñoz \& Torres (2014) en otras zonas del país, con docentes de educación primaria, en las cuales el profesorado tampoco logró identificar estos elementos en su formación inicial, y con el estudio de la Agencia de Calidad de la Educación (2016), en el que se destacó que el profesorado reconoció la falencia conceptual para abordar la formación ciudadana, así como también la falta de preparación metodológica para aprovechar las oportunidades que les presenta el currículum.

Los déficits en la formación inicial pueden ser evidenciados al hacer un análisis de las propias respuestas entregadas por las futuras educadoras, en donde existe cierta inseguridad, así como una simplicidad en el significado, la relevancia y la forma que abordan la temática en su trabajo cotidiano. Esto es consistente con los resultados de otras investigaciones anteriores que indagaron el rol del profesorado en la formación de nuevos ciudadanos, en las que se constaron una serie de desfases e incomprensiones, que finalmente terminaban por desvalorizar la importancia de la formación ciudadana en los primeros años de la educación formal (Reyes et al., 2013).

Lo señalado es especialmente importante si, además, se tiene presente que, en este proceso de desfase e incomprensión, las futuras educadoras se posicionan generalmente en una visión normativa y vertical de la educación ciudadana, lo que, por una parte, pone en evidencia la existencia de una tremenda brecha con la literatura especializada (Kerr, 1999) y, por otra, revela la existencia de una mirada reduccionista del rol que puede desempeñar la escuela (el jardín) en dicha educación. Esta situación repercute negativamente en la manera de abordar la enseñanza y aprendizaje de la ciudadanía en el aula infantil, en la medida en que predominan miradas adultocéntricas y un rol eminentemente pasivo de los estudiantes (Arrúe \& Consoli, 2010; Figueroa, 2016; García \& De Alba, 2014). Lo señalado nos indica la importancia que posee una adecuada educación ciudadana en la formación profesional inicial y, al mismo tiempo, pone de relieve la necesidad de repensar el rol que juega la educadora en la formación ciudadana de niños y niñas que asisten al jardín.

En relación con los resultados obtenidos, este rol está bastante apartado de las finalidades que presenta la educación ciudadana en el aula infantil y los objetivos que persigue la Ley 20911, pues se busca la formación 
desde la más tierna infancia de personas participativas, auténticas, criteriosas, empáticas y responsables, con poder de decisión y autonomía (Muñoz et al., 2019). Objetivos que, a la luz de los resultados obtenidos, parecen distantes, pues desde los discursos de las educadoras en formación se desprenden prácticas de enseñanza y aprendizaje alejadas de la comprensión del mundo y del entorno de los estudiantes, lo que incluso imposibilita el desarrollo activo del tema dentro de la propia comunidad educativa, como ha sido la tónica también en otras realidades (Graziella \& Rivera, 2016; Martínez \& Cumsille, 2015).

El significado que las futuras educadoras del estudio le atribuyen a la educación ciudadana se sitúa en el fomento de la identidad y de aquellos valores fundamentales que sustentan las buenas relaciones en el espacio educativo. Por ello, las experiencias relacionadas con la convivencia tienen una alta valoración, así como la promoción de la participación y del respeto entre niños y niñas, lo que reforzaría los valores para la vida democrática (Bolívar, 2007; 2016; Carrillo \& Jurado, 2017). Sin embargo, las futuras educadoras no hacen ese enlace ni refuerzan ese vínculo, sino que recurren a las Bases Curriculares del nivel (MINEDUC, 2001), al ámbito de formación personal y social (el núcleo de convivencia), porque es allí donde se alojan los aprendizajes relacionados con las normas, con los valores de la sociedad, la participación, la colaboración, la pertenencia y la diversidad, lo que les permite potenciar las habilidades sociales de niños y niñas (Gil-Madrona et al., 2016). No obstante, como hemos señalado, este vínculo con principios cívicos tan importantes jamás se hace desde una perspectiva ciudadana, sino desde una perspectiva normativa, desde un conjunto de reglas a cumplir, desde una disciplina prescrita o desde un currículum a trabajar, lo cual hace que estos valores pierdan proyección desde el punto de vista social, y se desvalorice su importancia y aporte en el mejoramiento de la convivencia ciudadana.

Finalmente, en relación con las estrategias utilizadas por las educadoras en el nivel parvulario, se identifican aquellas que emanan de las experiencias de diseño general para el aprendizaje, tales como el juego, la mediación y los proyectos de aula, las cuales se corresponden con los principios declarados en las Bases Curriculares (MINEDUC, 2001), los Estándares Pedagógicos de la Educación Parvularia (MINEDUC, 2012), el Marco para la Buena Enseñanza (MINEDUC, 2008), y con lo establecido en la Ley 20911, así como en tratados internacionales como la Declaración de los Derechos del Niño (ONU, 1959) y la Convención de los Derechos del Niño (ONU, 1989), por lo que, de manera particular, no se distinguen estrategias propias para la educación ciudadana. 


\section{Conclusiones}

A partir de la discusión de los resultados, las conclusiones permiten dar orientaciones de cómo abordar el desafío de la educación ciudadana en la formación inicial de las futuras educadoras y del profesorado en general.

En esta línea, es fundamental que la formación inicial docente proporcione experiencias y estrategias propias de educación ciudadana que permitan, al futuro profesorado, el diseño de la enseñanza y el aprendizaje en beneficio de una educación cívica, no solo centrada en la convivencia, sino capaz de formar desde una perspectiva crítica, reflexiva y propositiva, que facilite su desarrollo en el sistema educativo, y que a la vez impulse la participación, favoreciendo las interacciones entre quienes integran la comunidad educativa: niños, niñas y adolescentes, y entre estos con los adultos. Para ello, es primordial una perspectiva de colaboración, horizontalidad, diálogo y simetría que se pueda vivenciar en la formación inicial del profesorado.

Desde esta perspectiva, el círculo (estrategia que emerge en la investigación) es utilizado por las educadoras en formación de manera constante y sistemática, transformándose en un recurso esencial para abordar la ciudadanía, pues promueve la participación, genera reflexión y diálogo en una relación simétrica; aunque no es relacionado directamente con la educación ciudadana por las educadoras, sino más bien como una actividad complementaria. En esta línea, se hace necesario potenciar el círculo como estrategia que permite el aprendizaje de la ciudadanía en la educación infantil desde la mirada de los propios niños y niñas (Trilla \& Novella, 2011; Moliner et al., 2016), desplazando las visiones adultocéntricas que se podrían originar en este proceso (Figueroa, 2016), por lo que se presenta como un desafío a desarrollar para la educación ciudadana.

Asimismo, se hace necesario potenciar las experiencias formativas de las educadoras, tales como los talleres pedagógicos, que resultan muy significativos, ya que fomentan la reflexión y el diálogo, además de que posibilitan el planteamiento de temáticas emergentes relacionadas con la educación en contexto, siendo una instancia que contribuye a la vivencia de la ciudadanía.

También, las prácticas desarrolladas en el sistema educativo poseen una alta valoración por las futuras educadoras, pues contribuyen en la adquisición de experiencias que permitan afrontar la temática, posibilitándoles elaborar actividades de aprendizaje acordes (Castro-Zubizarreta \& GarcíaRuiz, 2016; Tejada-Fernández et al., 2017). Sin embargo, al no existir estrategias y orientaciones claras con respecto a la educación ciudadana, resulta 
especialmente preocupante que las experiencias por parte de las futuras educadoras en el sistema educativo puedan acrecentar modelos de ciudadanía focalizados en lo normativo, con un carácter pasivo y poco participativo. En este sentido, una de las limitaciones estaría dada por la exclusión de las apreciaciones de las educadoras mentoras en el sistema escolar, así como de las académicas formadoras, lo cual posibilitaría una mirada articulada del proceso.

Por lo anterior, se requiere definir orientaciones y estrategias que fomenten la educación ciudadana desde la formación inicial y que, de manera transversal, les den a las futuras educadoras la posibilidad de generar aprendizajes significativos y contextuales para la educación ciudadana, permitiéndoles abordar las problemáticas actuales en beneficio de una ciudadanía activa, participativa y democrática.

De este estudio emerge una línea importante de investigación, que se abre a partir de la promulgación de las nuevas Bases de Educación Parvularia (MINEDUC, 2018), las cuales reconocen la centralidad de niños y niñas como sujetos que deben ejercer sus derechos desde la primera infancia. Allí juega un rol relevante el núcleo de convivencia y ciudadanía, enfatizando aspectos relevantes como la inclusión, la diversidad social y las actitudes ciudadanas a partir de los primeros años. Estos énfasis se declaran de manera transversal en la trayectoria educativa de párvulos. Resulta necesario conocer cómo desde la formación inicial de educadoras y educadores se está interpretando e incorporando el ámbito de la educación ciudadana en este nivel, a fin de contribuir para que de manera efectiva las futuras educadoras y educadores puedan participar en el desarrollo de la ciudadanía desde la salacuna.

Finalmente, la investigación impulsa a indagar en otros aspectos que permitan avanzar en las estrategias, orientaciones e incorporación de la educación ciudadana en la formación inicial de las educadoras y del profesorado en general, ahondando en las experiencias de ciudadanía a partir de las disciplinas, planes de estudio, docentes y vivencias de práctica en los centros educativos, lo que posibilitará la articulación entre la formación inicial con los requerimientos contextuales del sistema educativo y las necesidades propias de niños, niñas y adolescentes, de modo que se les permita vivenciar la ciudadanía desde una perspectiva responsable, democrática y justa.

\section{Sobre los autores}

Olga Aurora Carrillo-Mardones es académica del Departamento de Ciencias de la Educación en la Facultad de Educación de la Universidad Católica de Temuco, Chile. Su área de investigación se enfoca al estudio de la formación 
ciudadana en el sistema educativo, la ciudadanía en las comunidades locales, la educación en valores y la perspectiva de género en educación.

Sixtina Pinochet-Pinochet es académica de la Escuela de Educación y miembro del Observatorio de Investigación Interdisciplinar en Educación para la Ciudadanía de la Universidad Católica del Norte, Chile. Sus áreas de investigación se focalizan en la enseñanza de la historia a partir de actores invisibilizados, el desarrollo de la educación para la ciudadanía en el contexto escolar y la formación inicial docente.

Carlos Muñoz-Labraña es académico e investigador de la Facultad de Educación de la Universidad de Concepción, Chile. Sus áreas de investigación se focalizan en la didáctica de las ciencias sociales, la educación ciudadana y su desarrollo en el sistema educativo.

Dorama Elisa Leal-Burgos es académica del Departamento de Infancia y Educación Básica de la Educación en la Facultad de Educación de la Universidad Católica de Temuco, Chile. Su área de investigación se enfoca en las prácticas reflexivas en la formación inicial docente.

\section{Referencias}

Agencia de la Calidad. (2018). Informe nacional ICCS 2016. MINEDUC. http:// archivos.agenciaeducacion.cl/ICCS_V03_22MARZO.pdf

Agencia de la Calidad de la Educación. (2016). Formación ciudadana en el sistema escolar chileno: una mirada a las prácticas actuales y recomendaciones de mejora. https://bibliotecadigital.mineduc.cl/bitstream/handle/ 20.500.12365/2174/mono-653.pdf? sequence=1\&isAllowed $=y$

Arrúe, C. \& Consoli, E. (2010). Espacios de infancia, ciudadanía y participación infantil [Actas del Congreso Internacional: Profundizando la democracia como forma de vida. Desafíos de la democracia participativa y los aprendizajes ciudadanos en el Siglo XXI. 13 al 16 de mayo de 2010 - Rosario, Argentina. Facultad de Ciencia Política y Relaciones Internacionales, Universidad Nacional de Rosario]. http://www.academia.edu/1128760/Espacios_de_infan cia_ciudadan\%C3\%ADa_y_participaci\%C3\%B3n_infantil

Bolívar, A. (2007). Educación para la ciudadanía. Algo más que una asignatura. Graó.

Bolívar, A. (2016). Educar democráticamente para una ciudadanía activa. Revista Internacional de Educación para la Justicia Social, 5(1), 69-87. https://doi. org/10.15366/riejs2016.5.1

Carrillo, O. \& Jurado, P. (2017). La educación técnico profesional y las competencias para la ciudadanía. El caso de las comunas de la Provincia de Concepción, Chile. Calidad en la Educación, 46, 133-164. http://dx.doi.org/10.4067/ S0718-45652017000100133

Castro-Zubizarreta, A. \& García-Ruiz, R. (2016). Vínculos entre familia y escuela: visión de los maestros en formación. magis, Revista Internacional de Investigación en Educación, 9(18), 193-208. http://dx.doi.org/10.11144/Javeriana. m9-18.vfev 
Coleman, H. \& Unrau, Y. (2005). Research and evaluation quantitative and qualitative approaches. En R. Grinnell \& Y. Unrau (Eds.). Social work (pp. 403420). Oxford University Press.

Consejo Asesor Presidencial contra los Conflictos de Interés, el Tráfico de Influencias y la Corrupción. (2015). Informe final. http://consejoanticorrupcion.cl/ wp-content/uploads/2015/04/Consejo_Anticorrupcion_Informe_Final.pdf

Cortina, A. (1997). Ciudadanos del Mundo. Hacia una teoría de la ciudadanía (3a ed.). Alianza Editorial.

Delors, J. (1996). La educación encierra un tesoro. Informe a la UNESCO de la comisión Internacional sobre la educación para el siglo XXI. Santillana/ UNESCO.

Díaz, M. \& Rojas, N. (2018). Educación para la ciudadanía en el posacuerdo. Revista Eleuthera, 20, 13-34. https://doi.org/10.17151/eleu.2019.20.2

Figueroa, C. (2016) ¿Ciudadanía de la niñez? Hallazgos de investigación sobre el movimiento por una cultura de derechos de la niñez y adolescencia en Chile. Última Década, 45, 118-139. http://dx.doi.org/10.4067/S071822362016000200007

García, F. \& De Alba, N. (2014). Città e cittadinanza nella scuola dell'infanzia. Un insegnamento per la formazione dei futuri maestri. Infanzia. Rivista di studi ed esperienze sull'educazione 0-6, 4/5, luglio-ottobre, monográfico "Educare alla cittadinanza attiva", 267-270. https://idus.us.es/handle/11441/28609

Gil-Madrona, P., Gómez-Barreto, I. \& González-Víllora, S. (2016). Percepción de los estudiantes de maestro de educación infantil sobre su formación intercultural. magis, Revista Internacional de Investigación en Educación, 9(18), 111-128. http://dx.doi.org/10.11144/Javeriana.m9-18.pmei

Gimeno, J. (2002). Educar y convivir en la cultura global: las exigencias de la ciudadanía. Morata.

Graziella, O. \& Rivera, J. (2016). Construcción de ciudadanía: la educación desde la infancia encaminada a la inclusión social. Tla-Melaua, Revista de Ciencias Sociales, 44, 52-71. http://dx.doi.org/10.32399/rtla.12.44.587

Hernández, R., Fernández, C. \& Baptista, P. (2006). Metodología de la investigación. McGraw-Hill.

Kerr, D. (1999). Citizenship Education: an International Comparison. Qualifications and Curriculum Authority.

Ley 20370 (2009). Establece la Ley General de Educación del Ministerio de Educación. Diario Oficial de la República de Chile, Santiago, 12 de septiembre.

Ley 20903 (2016). Crea el sistema de desarrollo profesional docente. Diario Oficial de la República de Chile, Santiago, 01 de abril.

Ley 20911 (2016). Crea el plan de formación ciudadana para los establecimientos educacionales reconocidos por el Estado. Diario oficial de la República de Chile, Santiago, 02 de abril.

Magendzo, A. (2008). Dilemas del currículum y la pedagogía. Analizando la reforma curricular desde una perspectiva crítica. Ediciones Lom.

Maiztegui, C. (2007). La participación como una opción transformadora en los procesos de educación ciudadana. REICE, 5(4), 144-160. https://www. redalyc.org/pdf/551/55140509.pdf

Martínez, M. \& Cumsille, P. (2015). La escuela como contexto de socialización política: influencias colectivas e individuales. En C. Cox \& J. Castillo (Eds.), 
Aprendizaje de la Ciudadanía. Contextos, experiencias y resultados (pp. 429457). Ediciones UC.

Mata, P. \& Ballesteros, B. (2011). Ciudadanía..., ¿cómo se aprende? Una investigación sobre discursos y experiencias de participación y transformación social. http://www.quadernsdigitals.net/index.php?accionMenu=hemeroteca. VisualizaArticulolU.visualiza\&articulo_id=11050\&PHPSESSID=2d7999983 8a38f990f8a4d470cc88189

MINEDUC. (2001). Bases Curriculares de la Educación Parvularia. Ministerio de Educación, República de Chile.

MINEDUC. (2008). Marco para la Buena Enseñanza (7a ed.). Ministerio de Educación, República de Chile.

MINEDUC. (2012). Estándares orientadores para las carreras de educación parvularia. Ministerio de Educación, República de Chile.

MINEDUC. (2018). Bases Curriculares de la Educación Parvularia. Subsecretaría de Educación Parvularia, Ministerio de Educación, República de Chile.

Moliner, O., Traver, J. A., Ruiz, M. A. \& Segarra, T. (2016). Estrategias que inciden en los procesos de democratización de la escuela. Una aproximación teórica. Revista Electrónica de Investigación Educativa, 18(2), 116-129. http:// redie.uabc.mx/redie/article/view/1110

Muñoz, C., Martínez, R., Muñoz-Grandón, C., Torres, B. \& Gutiérrez, K. (2019). Ciudadanizar la Escuela: de la Educación Cívica a la Formación Ciudadana. Un paso necesario y urgente en el sistema escolar. En C. Muñoz \& B. Torres (Eds.), Escuela y formación ciudadana, temas, escenarios y propuestas para su desarrollo (pp. 21-36). Universidad de Concepción.

Muñoz, C. \& Torres, B. (2014). La formación ciudadana en la escuela: problemas y desafíos. Revista Electrónica Educare, 2(18), 233-245. https://www.scielo. sa.cr/pdf/ree/v18n2/a12v18n2.pdf

Nussbaum, M. (2010). Sin fines de lucro. Por qué la democracia necesita las humanidades. Katz Editores.

Orellana, C. \& Muñoz, C. (2019). Escuela y formación ciudadana: Concepciones de ciudadanía, formación ciudadana y del rol de la escuela. Revista Electrónica Interuniversitaria de Formación del Profesorado, 22(2), 137-149. https:// doi.org/10.6018/reifop.22.2.370561

Osoro-Sierra, J. M. \& Castro-Zubizarreta, A. (2017). Educación y democracia: la escuela como "espacio" de participación. Revista Iberoamericana de Educación, 75(2), 89-108. https://rieoei.org/RIE/article/view/2635

Organización de Naciones Unidas. (1989) Asamblea General. Convención sobre los Derechos del Niño. Treaty Series, 1577, 3. https://www.refworld.org.es/ docid/50ac92492

ONU: Asamblea General. (20 de noviembre de 1959). Declaración de los Derechos del Niño. Autor.

Pagès, J. \& Santisteban, A. (2009). Una propuesta conceptual para la investigación en educación para la ciudadanía. Revista Educación y Pedagogía, 21(53), 15-31. https://www.google.com/url?sa=t\&rct=j\&q=\&esrc=s\&sou rce $=$ web\&cd $=\& v e d=2$ ahUKEwjrOszu1f3zAhUDQjABHYM_DvEQFnoECAc QAQ\&url=https\%3A\%2F\%2Frevistas.udea.edu.co\%2Findex.php\%2Frevist aeyp\%2Farticle\%2Fdownload\%2F9830\%2F9029\%2F\&usg=AOvVaw2K10 fCsp3KLogp6zss7fs3 
Peña, C. (2015). Escuela y vida cívica. En C. Cox \& J. Castillo (Eds.), Aprendizaje de la Ciudadanía. Contextos, experiencias y resultados (pp. 25-49). Ediciones UC.

Pérez, G. (2008). Investigación cualitativa: retos e interrogantes. Ediciones La Muralla.

Pinochet, S. (2015). Profesor, profesora: ¿Por qué los niños y las niñas no están en la historia? Concepciones del profesorado y del alumnado sobre la Historia de la infancia. [Tesis doctoral] Universidad Autónoma de Barcelona. http://www.tesisenred.net/bitstream/handle/10803/290848/sgpp1de1. pdf? sequence $=1$

Redón, S. (2010). La escuela como espacio de ciudadanía. Estudios Pedagógicos 36(2), 213-239. https://www.scielo.cl/scielo.php?script=sci_arttext\&pi $d=$ S0718-07052010000200013

Redón, S. (2019). Ciudadanía y educación parvularia: niñas y niños sujetos de derecho. Centro de investigación y educación inclusiva, Pontificia Universidad Católica de Valparaiso. http://eduinclusiva.cl/wp-content/uploads/2019/10/ DocSilvia.pdf

Reyes, L., Campos, J., Osandón, L. \& Muñoz, C. (2013). El profesorado y su rol en la formación de los nuevos ciudadanos: desfases entre las comprensiones, las actuaciones y las expectativas. Estudios Pedagógicos, 39(1), 217-237. https:// www.scielo.cl/scielo.php?pid=S0718-07052013000100013\&script=sci_arttext

Rodríguez, A., Flórez, R. \& Gómez, D. (2016). La formación en ciudadanía en escenarios de educación inicial: una experiencia con madres comunitarias. Panorama, 10(18), 102-119. https://doi.org/10.15765/pnrm.v10i18.826

Ruiz, L., Ñáñez, J. \& Robledo, C. (2016). La formación ciudadana en el nivel preescolar: una mirada comprensiva en instituciones educativas públicas de la ciudad de Ibagué. [Ponencia presentada en VII Coloquio Internacional de Educación - Universidad del Cauca]. http://www.unicauca.edu.co/eventos/ index.php/educoloquio/2016/paper/viewFile/490/303

Shuey, E. \& Kankaras, M. (2018). The power and promise of early learning. https://doi.org/10.1787/f9b2e53f-en

Stake, R. (2007). Investigación con estudio de caso. Morata.

Strauss, A. \& Corbin, J. (2002). Bases de la investigación cualitativa : técnicas y procedimientos para desarrollar la teoría fundamentada. Universidad de Antioquia.

Tejada-Fernández, J., Carvalho-Dias, M. \& Ruiz-Bueno, C. (2017). El prácticum en la formación de maestros: percepciones de los protagonistas. magis, Revista Internacional de Investigación en Educación, 9(19), 91-114. https:// doi.org/10.11144/Javeriana.m9-19.pfmp

Trilla, J. \& Novella, A. (2001). Educación y participación social de la infancia. Revista Iberoamericana de Educación, 26, 137-164. https://rieoei.org/historico/ documentos/rie26a07.htm

Trilla, J. \& Novella, A. (2011). Participación, democracia y formación para la ciudadanía. Los consejos de la infancia. Revista de Educación, 356, 23-43. http:// www.revistaeducacion.educacion.es/re2011/re2011_02.pdf 